\title{
Almost Periodic Solution of a Discrete Schoener's Competition Model with Delays
}

\author{
Hui Zhang, Yingqi Li, Bin Jing, Xiaofeng Fang, and Jing Wang \\ Mathematics and OR Section, Xi'an Research Institute of High-Tech Hongqing Town, Xi'an, Shaanxi 710025, China \\ Correspondence should be addressed to Hui Zhang; zh53054958@163.com
}

Received 8 June 2014; Accepted 12 July 2014; Published 24 July 2014

Academic Editor: Cengiz Çinar

Copyright (C) 2014 Hui Zhang et al. This is an open access article distributed under the Creative Commons Attribution License, which permits unrestricted use, distribution, and reproduction in any medium, provided the original work is properly cited.

We consider an almost periodic discrete Schoener's competition model with delays. By means of an almost periodic functional hull theory and constructing a suitable Lyapunov function, sufficient conditions are obtained for the existence of a unique strictly positive almost periodic solution which is globally attractive. An example together with numerical simulation indicates the feasibility of the main result.

\section{Introduction}

In 2009, Wu et al. [1] had studied a discrete Schoener's competition mode with delays:

$$
\begin{aligned}
& x_{1}(n+1) \\
& \begin{aligned}
&=x_{1}(n) \exp \left[\frac{a_{10}(n)}{x_{1}\left(n-\tau_{10}\right)+k_{1}(n)}-a_{11}(n) x_{1}\left(n-\tau_{11}\right)\right. \\
&\left.-a_{12}(n) x_{2}\left(n-\tau_{12}\right)-c_{1}(n)\right], \\
& x_{2}(n+1) \\
&=x_{2}(n) \exp \left[\frac{a_{20}(n)}{x_{2}\left(n-\tau_{20}\right)+k_{2}(n)}-a_{21}(n) x_{1}\left(n-\tau_{21}\right)\right. \\
& \\
&\left.\quad-a_{22}(n) x_{2}\left(n-\tau_{22}\right)-c_{2}(n)\right],
\end{aligned}
\end{aligned}
$$

where $\left\{k_{i}(n)\right\},\left\{a_{i j}(n)\right\}$, and $\left\{c_{i}(n)\right\}$ are real positive bounded sequences and $\tau_{i j}$ are positive integers, $i=1,2 ; j=0,1,2$. Sufficient conditions which guarantee the permanence and the global attractivity of positive solutions for system (1) are obtained.
By the biological meaning, the system (1) is considered together with the following initial condition:

$$
\begin{aligned}
& x_{i}(s)=\varphi_{i}(s) \geq 0, \quad \varphi_{i}(0)>0, \\
& s \in[-\tau, 0] \cap Z=\{-\tau,-\tau+1, \ldots, 0\}, \quad i=1,2,
\end{aligned}
$$

where $\tau=\max _{1 \leq i \leq 2,0 \leq j \leq 2}\left\{\tau_{i j}\right\}$. Let $\left(x_{1}(n), x_{2}(n)\right)$ be any solution of system (1) with the initial condition (2). One could easily see that $x_{i}(n)>0, i=1,2$ for all $n \in N$.

Schoener's competition system has been studied by many scholars. Topics such as existence, uniqueness, and global attractivity of positive periodic solutions of the system were extensively investigated, and many excellent results have been derived (see [1-6] and the references cited therein). Recently, a few papers investigate the global stability of the pure-delay model (see [7-13]).

Notice that the investigation of almost periodic solutions for difference equations is one of most important topics in the qualitative theory of difference equations due to the applications in biology, ecology, neural network, and so forth (see [14-21] and the references cited therein). But to the best of the author's knowledge, to this day, still no scholars have studied the almost periodic version which is corresponding to system (1). Therefore, with stimulation from the works of $[12,19]$, the main purpose of this paper is to derive a set of sufficient conditions ensuring the existence of a unique 
strictly positive almost periodic solution of system (1) which is globally attractive.

Denote by $Z$ and $Z^{+}$the set of integers and the set of nonnegative integers, respectively. For any bounded sequence $\{f(n)\}$ defined on $Z$, define $f^{u}=\sup _{n \in Z} f(n), f^{l}=$ $\inf _{n \in Z} f(n)$.

Throughout this paper, we assume the following.

(H1) $k_{i}(n), a_{i j}(n)$, and $c_{i}(n)$ are bounded positive almost periodic sequences such that

$$
\begin{aligned}
& 0<k_{i}^{l} \leq k_{i}(n) \leq k_{i}^{u}, \\
& 0<a_{i j}^{l} \leq a_{i j}(n) \leq a_{i j}^{u}, \\
& 0<c_{i}^{l} \leq c_{i}(n) \leq c_{i}^{u}, \\
& i=1,2, \quad j=0,1,2 .
\end{aligned}
$$

The remaining part of this paper is organized as follows. In Section 2, we will introduce some definitions and several useful lemmas. In Section 3, by applying the theory of difference inequality, we present the permanence results for system (1). In Section 4, we establish the sufficient conditions for the existence of a unique globally attractive almost periodic solution of system (1). The main result is illustrated by an example with a numerical simulation in the last section.

\section{Preliminaries}

In this section, we give the definitions and lemmas of the terminologies involved.

Definition 1 (see [22]). A sequence $x: Z \rightarrow R$ is called an almost periodic sequence if the $\varepsilon$-translation set of $x$

$$
E\{\varepsilon, x\}=\{\tau \in Z:|x(n+\tau)-x(n)|<\varepsilon, \forall n \in Z\}
$$

is a relatively dense set in $Z$ for all $\varepsilon>0$; that is, for any given $\varepsilon>0$, there exists an integer $l(\varepsilon)>0$ such that each interval of length $l(\varepsilon)$ contains an integer $\tau \in E\{\varepsilon, x\}$ with

$$
|x(n+\tau)-x(n)|<\varepsilon, \quad \forall n \in Z .
$$

$\tau$ is called an $\varepsilon$-translation number of $x(n)$.

Definition 2 (see [23]). Let $D$ be an open subset of $R^{m}, f: Z \times$ $D \rightarrow R^{m} \cdot f(n, x)$ is said to be almost periodic in $n$ uniformly for $x \in D$ if for any $\varepsilon>0$, and any compact set $S \subset D$, there exists a positive integer $l=l(\varepsilon, S)$ such that any interval of length $l$ contains an integer $\tau$ for which

$$
|f(n+\tau, x)-f(n, x)|<\varepsilon, \quad \forall(n, x) \in Z \times S .
$$

$\tau$ is called an $\varepsilon$-translation number of $f(n, x)$.

Definition 3 (see [24]). The hull of $f$, denoted by $H(f)$, is defined by

$$
\begin{aligned}
H(f)=\left\{g(n, x): \lim _{k \rightarrow \infty} f\left(n+\tau_{k}, x\right)\right. & \\
= & g(n, x) \text { uniformly on } Z \times S\},
\end{aligned}
$$

for some sequence $\left\{\tau_{k}\right\}$, where $S$ is any compact set in $D$.
Definition 4. Suppose that $X(n)=\left(x_{1}(n), x_{2}(n)\right)$ is any solution of system (1). $X(n)$ is said to be a strictly positive solution in $Z$ if

$$
0<\inf _{n \in Z} x_{i}(n) \leq \sup _{n \in Z} x_{i}(n)<\infty, \quad i=1,2,
$$

for $n \in Z$.

Lemma 5 (see $[25])$. Assume that $\{x(n)\}$ satisfies $x(n)>0$ and

$$
x(n+1) \leq x(n) \exp \{a(n)-b(n) x(n)\}
$$

for $n \in N$, where $a(n)$ and $b(n)$ are nonnegative sequences bounded above and below by positive constants. Then

$$
\limsup _{n \rightarrow+\infty} x(n) \leq \frac{1}{b^{l}} \exp \left\{a^{u}-1\right\}
$$

Lemma 6 (see [25]). Assume that $\{x(n)\}$ satisfies

$$
\begin{gathered}
x(n+1) \geq x(n) \exp \{a(n)-b(n) x(n)\}, \quad n \geq N_{0}, \\
\limsup _{n \rightarrow+\infty} x(n) \leq x^{*},
\end{gathered}
$$

and $x\left(N_{0}\right)>0$, where $a(n)$ and $b(n)$ are nonnegative sequences bounded above and below by positive constants and $N_{0} \in N$. Then

$$
\liminf _{n \rightarrow+\infty} x(n) \geq \min \left\{\frac{a^{l}}{b^{u}} \exp \left\{a^{l}-b^{u} x^{*}\right\}, \frac{a^{l}}{b^{u}}\right\} .
$$

\section{Permanence and Global Attractivity}

Now we state several lemmas which will be useful in proving our main result.

Proposition 7 (see [1]). Any solution $\left(x_{1}(n), x_{2}(n)\right)$ of system (1) with the initial condition (2) is positive and ultimately bounded; that is,

$$
\limsup _{n \rightarrow+\infty} x_{i}(n) \leq M_{i}
$$

where

$$
M_{i}=\frac{1}{a_{i i}^{l}} \exp \left\{\frac{a_{i 0}^{u}}{k_{i}^{l}}\left(\tau_{i i}+1\right)-1\right\}, \quad i=1,2 .
$$

Proposition 8. Assume that

$$
\begin{aligned}
& \frac{a_{10}^{l}}{M_{1}+k_{1}^{u}}-a_{12}^{u} M_{2}-c_{1}^{u}>0 \\
& \frac{a_{20}^{l}}{M_{2}+k_{2}^{u}}-a_{21}^{u} M_{1}-c_{2}^{u}>0
\end{aligned}
$$

holds; then for any solution $\left(x_{1}(n), x_{2}(n)\right)$ of system (1) with the initial condition (2), one has

$$
\liminf _{n \rightarrow+\infty} x_{i}(n) \geq m_{i}, \quad i=1,2,
$$

where $m_{1}$ and $m_{2}$ are defined by (24) and (26), respectively. 
Proof. For any small positive constant $\varepsilon>0$, according to Proposition 7, there exists a $N_{1}>0$ such that, for all $n>N_{1}$ and $i=1,2$,

$$
x_{i}(n) \leq M_{i}+\varepsilon .
$$

It follows from (17) and the first equation of system (1), for $n \geq N_{1}+\tau$,

$$
\begin{aligned}
& x_{1}(n+1) \\
& \geq x_{1}(n) \exp \left[\frac{a_{10}^{l}}{\left(M_{1}+\varepsilon\right)+k_{1}^{u}}-a_{11}^{u}\left(M_{1}+\varepsilon\right)\right. \\
& \left.\quad-a_{12}^{u}\left(M_{2}+\varepsilon\right)-c_{1}^{u}\right] \triangleq x_{1}(n) \exp \left\{A_{1}^{\varepsilon}\right\},
\end{aligned}
$$

where $A_{1}^{\varepsilon}=\left(a_{10}^{l} /\left(\left(M_{1}+\varepsilon\right)+k_{1}^{u}\right)\right)-a_{11}^{u}\left(M_{1}+\varepsilon\right)-a_{12}^{u}\left(M_{2}+\varepsilon\right)-c_{1}^{u}$.

Thus, by using (18) we obtain

$$
x_{1}\left(n-\tau_{11}\right) \leq x_{1}(n) \exp \left\{-\tau_{11} A_{1}^{\varepsilon}\right\} .
$$

Substituting (19) into the first equation of system (1), for $n \geq$ $N_{1}+\tau_{11}$, it follows that

$$
\begin{aligned}
& x_{1}(n+1) \\
& \qquad x_{1}(n) \exp \left[\frac{a_{10}^{l}}{\left(M_{1}+\varepsilon\right)+k_{1}^{u}}-a_{12}^{u}\left(M_{2}+\varepsilon\right)\right. \\
& \\
& \left.\quad-c_{1}^{u}-a_{11}(n) \exp \left\{-\tau_{11} A_{1}^{\varepsilon}\right\} x_{1}(n)\right] .
\end{aligned}
$$

When $\varepsilon$ is an arbitrary small positive constant, it follows from condition ( $\mathrm{H} 2)$

$$
\frac{a_{10}^{l}}{\left(M_{1}+\varepsilon\right)+k_{1}^{u}}-a_{12}^{u}\left(M_{2}+\varepsilon\right)-c_{1}^{u}>0 .
$$

Thus, as a direct corollary of Lemma 6, according to (14) and (20), one has

$$
\liminf _{n \rightarrow+\infty} x_{1}(n) \geq \min \left\{B_{1 \varepsilon}, B_{2 \varepsilon}\right\},
$$

where

$$
\begin{gathered}
B_{1 \varepsilon}=\frac{1}{a_{11}^{u}}\left[\frac{a_{10}^{l}}{\left(M_{1}+\varepsilon\right)+k_{1}^{u}}-a_{12}^{u}\left(M_{2}+\varepsilon\right)-c_{1}^{u}\right] \exp \left\{\tau_{11} A_{1}^{\varepsilon}\right\}, \\
B_{2 \varepsilon}=B_{1 \varepsilon} \exp \left\{\frac{a_{10}^{l}}{\left(M_{1}+\varepsilon\right)+k_{1}^{u}}-a_{12}^{u}\left(M_{2}+\varepsilon\right)\right. \\
\left.-c_{1}^{u}-M_{1} a_{11}^{u} \exp \left\{-\tau_{11} A_{1}^{\varepsilon}\right\}\right\} .
\end{gathered}
$$

Letting $\varepsilon \rightarrow 0$, it follows that

$$
\liminf _{n \rightarrow+\infty} x_{1}(n) \geq \frac{1}{2} \min \left\{B_{1}, B_{2}\right\} \triangleq m_{1}>0
$$

where

$$
\begin{gathered}
B_{1}=\frac{1}{a_{11}^{u}}\left[\frac{a_{10}^{l}}{M_{1}+k_{1}^{u}}-a_{12}^{u} M_{2}-c_{1}^{u}\right] \exp \left\{\tau_{11} A_{1}\right\}, \\
B_{2}=B_{1} \exp \left\{\frac{a_{10}^{l}}{M_{1}+k_{1}^{u}}-a_{12}^{u} M_{2}\right. \\
\left.-c_{1}^{u}-M_{1} a_{11}^{u} \exp \left\{-\tau_{11} A_{1}\right\}\right\}, \\
A_{1}=\frac{a_{10}^{l}}{M_{1}+k_{1}^{u}}-a_{11}^{u} M_{1}-a_{12}^{u} M_{2}-c_{1}^{u} .
\end{gathered}
$$

Similar to the analysis of (18)-(24), by applying (17), from the second equation of system (1), we also have that

$$
\liminf _{n \rightarrow+\infty} x_{2}(n) \geq \frac{1}{2} \min \left\{D_{1}, D_{2}\right\} \triangleq m_{2}>0,
$$

where

$$
\begin{gathered}
D_{1}=\frac{1}{a_{22}^{u}}\left[\frac{a_{20}^{l}}{M_{2}+k_{2}^{u}}-a_{21}^{u} M_{1}-c_{2}^{u}\right] \exp \left\{\tau_{22} C_{1}\right\}, \\
D_{2}=D_{1} \exp \left\{\frac{a_{20}^{l}}{M_{2}+k_{2}^{u}}-a_{21}^{u} M_{1}-c_{2}^{u}\right. \\
\left.\quad-M_{2} a_{22}^{u} \exp \left\{-\tau_{22} C_{1}\right\}\right\}, \\
C_{1}=\frac{a_{20}^{l}}{M_{2}+k_{2}^{u}}-a_{22}^{u} M_{2}-a_{21}^{u} M_{1}-c_{2}^{u} .
\end{gathered}
$$

The proof is completed.

Note that condition (H2) of Proposition 8 is weakened compared to condition $(\mathrm{H})$ in $[1]$.

Theorem 9 (see [1]). Assume that (H2) holds; system (1) is permanent.

Proposition 10 (see [1]). Assume that (H2) holds and further that there exist positive constants $\alpha_{1}, \alpha_{2}$, and $\eta>0$ such that

$$
\alpha_{i} E_{i i}-\left(\alpha_{i} F_{i j}+\alpha_{j} G_{j i}\right) \geq \eta, \quad i, j=1,2, i \neq j
$$

where

$$
\begin{aligned}
E_{i i}= & \min \left(a_{i i}^{l}, \frac{2}{M_{i}}-a_{i i}^{u}\right)-\frac{a_{i 0}^{u}}{\left(k_{i}^{l}\right)^{2}}\left(1+\tau_{i i} a_{i i}^{u} M_{i} B_{i}^{u}\right) \\
& -\tau_{i i}\left(a_{i i}^{u}\right)^{2} M_{i} B_{i}^{u}, \\
F_{i j}= & \tau_{i i} a_{i i}^{u} A_{i}^{u}\left(\frac{a_{i 0}^{u}}{k_{i}^{l}}+a_{i i}^{u} M_{i}+a_{i j}^{u} M_{j}+c_{i}^{u}\right), \\
G_{j i}= & a_{j i}^{u}+\tau_{j j} a_{j j}^{u} M_{j} B_{j}^{u} a_{j i}^{u}, \quad i, j=1,2 ; i \neq j,
\end{aligned}
$$




$$
\begin{gathered}
A_{i}(s)=\exp \left\{\theta _ { i } ( s ) \left(\frac{a_{i 0}(s)}{x_{i}^{*}\left(s-\tau_{i 0}\right)+k_{i}(s)}-a_{i i} x_{i}^{*}\left(s-\tau_{i i}\right)\right.\right. \\
\left.\left.-a_{i j} x_{j}^{*}\left(s-\tau_{i j}\right)-c_{i}(s)\right)\right\}, \\
B_{i}(s)=\exp \left\{\varphi _ { i } ( s ) \left(\frac{a_{i 0}(s)}{x_{i}\left(s-\tau_{i 0}\right)+k_{i}(s)}-a_{i i} x_{i}\left(s-\tau_{i i}\right)\right.\right. \\
\left.-a_{i j} x_{j}\left(s-\tau_{i j}\right)-c_{i}(s)\right) \\
+\left(1-\varphi_{i}(s)\right)\left(\frac{a_{i 0}(s)}{x_{i}^{*}\left(s-\tau_{i 0}\right)+k_{i}(s)}\right. \\
-a_{i i} x_{i}^{*}\left(s-\tau_{i i}\right) \\
\left.\left.-a_{i j} x_{j}^{*}\left(s-\tau_{i j}\right)-c_{i}(s)\right)\right\},
\end{gathered}
$$

$\theta_{i}(s), \varphi_{i}(s) \in(0,1), i, j=1,2, i \neq j$. Then system (1) is globally attractive; that is, for any two positive solutions $\left(x_{1}(n), x_{2}(n)\right)$ and $\left(x_{1}^{*}(n), x_{2}^{*}(n)\right)$ of system $(1)$, we have

$$
\lim _{n \rightarrow+\infty}\left(x_{i}^{*}(n)-x_{i}(n)\right)=0, \quad i=1,2 .
$$

\section{Almost Periodic Solution}

In this section, by means of an almost periodic functional hull theory and constructing a suitable Lyapunov function, we will study the existence of a globally attractive almost periodic solution of system (1) with initial condition (2) and obtain the sufficient conditions.

Let $\left\{\delta_{k}\right\}$ be any integer valued sequence such that $\delta_{k} \rightarrow$ $\infty$ as $k \rightarrow \infty$. According to Lemma 5 , taking a subsequence if necessary, we have $k_{i}\left(n+\delta_{k}\right) \rightarrow k_{i}^{*}(n), a_{i j}\left(n+\delta_{k}\right) \rightarrow$ $a_{i j}^{*}(n), c_{i}\left(n+\delta_{k}\right) \rightarrow c_{i}^{*}(n), i=1,2, j=0,1,2$, as $k \rightarrow \infty$ for $n \in Z$. Then we get a hull equation of system (1) as follows:

$$
\begin{aligned}
& x_{1}(n+1) \\
& =x_{1}(n) \exp \left[\frac{a_{10}^{*}(n)}{x_{1}\left(n-\tau_{10}\right)+k_{1}^{*}(n)}-a_{11}^{*}(n) x_{1}\left(n-\tau_{11}\right)\right. \\
& \left.-a_{12}^{*}(n) x_{2}\left(n-\tau_{12}\right)-c_{1}^{*}(n)\right] \text {, } \\
& x_{2}(n+1) \\
& \begin{array}{c}
=x_{2}(n) \exp \left[\frac{a_{20}^{*}(n)}{x_{2}\left(n-\tau_{20}\right)+k_{2}^{*}(n)}-a_{21}^{*}(n) x_{1}\left(n-\tau_{21}\right)\right. \\
\left.-a_{22}^{*}(n) x_{2}\left(n-\tau_{22}\right)-c_{2}^{*}(n)\right] .
\end{array}
\end{aligned}
$$

By the almost periodic theory, we can conclude that if system (1) satisfies (H2) and (H3), then the hull equation (31) of system (1) also satisfies (H2) and (H3).
By Theorem 3.4 in [26], we can easily obtain the lemma as follows.

Lemma 11. If each hull equation of system (1) has a unique strictly positive solution, then the almost periodic difference system (1) has a unique strictly positive almost periodic solution.

Now we investigate a globally attractive almost periodic solution of system (1).

Theorem 12. If the almost periodic difference system (1) satisfies (H1), (H2), and (H3), then the almost periodic difference system (1) admits a unique strictly positive almost periodic solution, which is globally attractive.

Proof. By Lemma 11, we only need to prove that each hull equation of system (1) has a unique globally attractive almost periodic sequence solution; hence we firstly prove that each hull equation of system (1) has at least one strictly positive solution (the existence) and then we prove that each hull equation of system (1) has a unique strictly positive solution (the uniqueness).

Now we prove the existence of a strictly positive solution of any hull equation (31). By the almost periodicity of $\left\{k_{i}(n)\right\},\left\{a_{i j}(n)\right\}$, and $\left\{c_{i}(n)\right\}, i=1,2, j=0,1,2$, there exists an integer valued sequence $\left\{\tau_{k}\right\}$ with $\tau_{k} \rightarrow \infty$ as $k \rightarrow \infty$ such that $k_{i}^{*}\left(n+\tau_{k}\right) \rightarrow k_{i}^{*}(n), a_{i j}^{*}\left(n+\tau_{k}\right) \rightarrow a_{i j}^{*}(n), c_{i}^{*}\left(n+\tau_{k}\right) \rightarrow$ $c_{i}^{*}(n), i=1,2, j=0,1,2$, as $k \rightarrow \infty$ for $n \in Z$. Suppose that $X=\left(x_{1}(n), x_{2}(n)\right)$ is any solution of hull equation (31). By the proof of Propositions 7 and 8, we have

$$
m_{i} \leq \liminf _{n \rightarrow+\infty} x_{i}(n) \leq \limsup _{n \rightarrow+\infty} x_{i}(n) \leq M_{i}, \quad i=1,2 .
$$

And also

$$
0<\inf _{n \in Z^{+}} x_{i}(n) \leq \sup _{n \in Z^{+}} x_{i}(n)<\infty, \quad i=1,2 .
$$

Let $\varepsilon$ be an arbitrary small positive number. It follows from (32) that there exists a positive integer $n_{0}$ such that $m_{i}-\varepsilon \leq x_{i}(n) \leq M_{i}+\varepsilon, n \geq n_{0}, i=1,2$. Write $X_{k}(n)=$ $X\left(n+\tau_{k}\right)=\left(x_{1 k}(n), x_{2 k}(n)\right)$, for all $n \geq n_{0}+\tau-\tau_{k}, k \in Z^{+}$. We claim that there exists a sequence $\left\{y_{i}(n)\right\}$, and a subsequence of $\left\{\tau_{k}\right\}$, which we still denote by $\left\{\tau_{k}\right\}$ such that $x_{i k}(n) \rightarrow y_{i}(n)$ uniformly in $n$ on any finite subset $B$ of $Z$ as $k \rightarrow \infty$, where $B=\left\{a_{1}, a_{2}, \ldots, a_{m}\right\}, a_{h} \in Z(h=1,2, \ldots, m)$, and $m$ is a finite number.

In fact, for any finite subset $B \subset Z$, when $k$ is large enough, $\tau_{k}+a_{h}-\tau>n_{0}, h=1,2, \ldots, m$. So

$$
m_{i}-\varepsilon \leq x_{i}\left(n+\tau_{k}\right) \leq M_{i}+\varepsilon, \quad i=1,2 ;
$$

that is, $\left\{x_{i}\left(n+\tau_{k}\right)\right\}$ are uniformly bounded for $k$ large enough.

Now, for $a_{1} \in B$, we can choose a subsequence $\left\{\tau_{k}^{(1)}\right\}$ of $\left\{\tau_{k}\right\}$ such that $\left\{x_{i}\left(a_{1}+\tau_{k}^{(1)}\right)\right\}$ uniformly converges on $Z^{+}$for $k$ large enough.

Similarly, for $a_{2} \in B$, we can choose a subsequence $\left\{\tau_{k}^{(2)}\right\}$ of $\left\{\tau_{k}^{(1)}\right\}$ such that $\left\{x_{i}\left(a_{2}+\tau_{k}^{(2)}\right)\right\}$ uniformly converges on $Z^{+}$ for $k$ large enough.

Repeating this procedure, for $a_{m} \in B$, we can choose a subsequence $\left\{\tau_{k}^{(m)}\right\}$ of $\left\{\tau_{k}^{(m-1)}\right\}$ such that $\left\{x_{i}\left(a_{m}+\tau_{k}^{(m)}\right)\right\}$ uniformly converges on $Z^{+}$for $k$ large enough. 
Now pick the sequence $\left\{\tau_{k}^{(m)}\right\}$ which is a subsequence of $\left\{\tau_{k}\right\}$, which we still denote by $\left\{\tau_{k}\right\}$, then for all $n \in B$, we have $x_{i}\left(n+\tau_{k}\right) \rightarrow y_{i}(n)$ uniformly in $n \in B$, as $k \rightarrow \infty$.

By the arbitrary of $B$, the conclusion is valid.

Combining with

$x_{1 k}(n+1)$

$$
\begin{aligned}
&=x_{1 k}(n) \exp \left[\frac{a_{10}^{*}\left(n+\tau_{k}\right)}{x_{1 k}\left(n-\tau_{10}\right)+k_{1}^{*}\left(n+\tau_{k}\right)}\right. \\
& \quad-a_{11}^{*}\left(n+\tau_{k}\right) x_{1 k}\left(n-\tau_{11}\right) \\
&\left.\quad-a_{12}^{*}\left(n+\tau_{k}\right) x_{2 k}\left(n-\tau_{12}\right)-c_{1}^{*}\left(n+\tau_{k}\right)\right],
\end{aligned}
$$

$x_{2 k}(n+1)$

$$
\begin{aligned}
&=x_{2 k}(n) \exp \left[\frac{a_{20}^{*}\left(n+\tau_{k}\right)}{x_{2 k}\left(n-\tau_{20}\right)+k_{2}^{*}\left(n+\tau_{k}\right)}\right. \\
&-a_{21}^{*}\left(n+\tau_{k}\right) x_{1 k}\left(n-\tau_{21}\right) \\
&\left.\quad-a_{22}^{*}\left(n+\tau_{k}\right) x_{2 k}\left(n-\tau_{22}\right)-c_{2}^{*}\left(n+\tau_{k}\right)\right],
\end{aligned}
$$

gives

$$
\begin{aligned}
& y_{1}(n+1) \\
& =y_{1}(n) \exp \left[\frac{a_{10}^{*}(n)}{y_{1}\left(n-\tau_{10}\right)+k_{1}^{*}(n)}-a_{11}^{*}(n) y_{1}\left(n-\tau_{11}\right)\right. \\
& \left.\quad-a_{12}^{*}(n) y_{2}\left(n-\tau_{12}\right)-c_{1}^{*}(n)\right], \\
& \begin{aligned}
y_{2}(n+1) \\
=y_{2}(n) \exp \left[\frac{a_{20}^{*}(n)}{y_{2}\left(n-\tau_{20}\right)+k_{2}^{*}(n)}-a_{21}^{*}(n) y_{1}\left(n-\tau_{21}\right)\right. \\
\\
\left.-a_{22}^{*}(n) y_{2}\left(n-\tau_{22}\right)-c_{2}^{*}(n)\right] .
\end{aligned}
\end{aligned}
$$

We can easily see that $Y(n)=\left(y_{1}(n), y_{2}(n)\right)$ is a solution of hull equation (31) and $m_{i}-\varepsilon \leq y_{i}(n) \leq M_{i}+\varepsilon$, $i=1$, 2, for $n \in Z$. Since $\varepsilon$ is an arbitrary small positive number, it follows that $m_{i} \leq y_{i}(n) \leq M_{i}, i=1,2$, for $n \in Z$; that is,

$$
0<\inf _{n \in Z} y_{i}(n) \leq \sup _{n \in Z} y_{i}(n)<\infty, \quad i=1,2 .
$$

Hence each hull equation of almost periodic difference system (1) has at least one strictly positive solution.

Now we prove the uniqueness of the strictly positive solution of each hull equation (31). Suppose that the hull equation (31) has two arbitrary strictly positive solutions $\left(x_{1}^{*}(n), x_{2}^{*}(n)\right)$ and $\left(y_{1}^{*}(n), y_{2}^{*}(n)\right)$. We construct a Lyapunov functional

$$
V^{*}(n)=\sum_{i=1}^{2} \beta_{i}\left(V_{i 1}^{*}(n)+V_{i 2}^{*}(n)+V_{i 3}^{*}(n)\right), \quad n \in Z,
$$

where

$$
\begin{aligned}
& V_{i 1}^{*}(n)=\left|\ln x_{i}^{*}(n)-\ln y_{i}^{*}(n)\right| \\
& V_{i 2}^{*}(n) \\
& =\sum_{s=n-\tau_{i 0}}^{n-1} \frac{a_{i 0}\left(s+\tau_{i 0}\right)}{k_{i}^{2}\left(s+\tau_{i 0}\right)}\left|x_{i}^{*}(s)-y_{i}^{*}(s)\right| \\
& +\sum_{s=n-\tau_{i j}}^{n-1} a_{i j}\left(s+\tau_{i j}\right)\left|x_{j}^{*}(s)-y_{j}^{*}(s)\right| \\
& +\sum_{s=n}^{n-1+\tau_{i i}} a_{i i}(s) \\
& \times \sum_{u=s-\tau_{i i}}^{n-1}\left\{A _ { i } ( u ) \left[\frac{a_{i 0}(u)}{k_{i}(u)}+a_{i i}(u) M_{i}\right.\right. \\
& \left.+a_{i j}(u) M_{j}+c_{i}(u)\right] \\
& \times\left|x_{i}^{*}(u)-y_{i}^{*}(u)\right| \\
& +M_{i} B_{i}(u) \frac{a_{i 0}(u)}{k_{i}^{2}(u)} \\
& \times\left|x_{i}^{*}\left(u-\tau_{i 0}\right)-y_{i}^{*}\left(u-\tau_{i 0}\right)\right| \\
& +M_{i} B_{i}(u) a_{i i}(u) \\
& \times\left|x_{i}^{*}\left(u-\tau_{i i}\right)-y_{i}^{*}\left(u-\tau_{i i}\right)\right| \\
& +M_{i} B_{i}(u) a_{i j}(u) \\
& \left.\times\left|x_{j}^{*}\left(u-\tau_{i j}\right)-y_{j}^{*}\left(u-\tau_{i j}\right)\right|\right\} \text {, }
\end{aligned}
$$

$$
\begin{aligned}
V_{i 3}^{*}(n)=M_{i} \sum_{l=n-\tau_{i 0}}^{n-1} \frac{a_{i 0}\left(l+\tau_{i 0}\right)}{k_{i}^{2}\left(l+\tau_{i 0}\right)} B_{i}\left(l+\tau_{i 0}\right) \\
\quad \times\left|x_{j}^{*}(l)-y_{j}^{*}(l)\right| \sum_{s=l+\tau_{i 0}+1}^{l+\tau_{i 0}+\tau_{i i}} a_{i i}(s) \\
+M_{i} \sum_{l=n-\tau_{i i}}^{n-1} B_{i}\left(l+\tau_{i i}\right) a_{i i}\left(l+\tau_{i i}\right) \\
\quad \times\left|x_{i}^{*}(l)-y_{i}^{*}(l)\right| \sum_{s=l+\tau_{i i}+1}^{l+2 \tau_{i i}} a_{i i}(s)
\end{aligned}
$$




$$
\begin{array}{r}
+M_{i} \sum_{l=n-\tau_{i j}}^{n-1} B_{i}\left(l+\tau_{i j}\right) a_{i j}\left(l+\tau_{i j}\right) \\
\times\left|x_{j}^{*}(l)-y_{j}^{*}(l)\right| \sum_{s=l+\tau_{i j}+1}^{l+\tau_{i j}+\tau_{i i}} a_{i i}(s), \\
i, j=1,2, \quad i \neq j .
\end{array}
$$

Calculating the difference of $V^{*}(n)$ along the solution of the hull equation (31), one has

$$
\Delta V^{*}(n) \leq-\eta \sum_{i=1}^{2}\left|x_{i}^{*}(n)-y_{i}^{*}(n)\right|, \quad n \in Z .
$$

From (40), we can see that $V^{*}(n)$ is a nonincreasing function on $Z$. Summing both sides of the above inequalities from $n$ to 0 , we have

$$
\eta \sum_{k=n}^{0} \sum_{i=1}^{2}\left|x_{i}^{*}(k)-y_{i}^{*}(k)\right| \leq V^{*}(0)-V^{*}(n+1)
$$

$n<0$.

Note that $V^{*}(n)$ is bounded. Hence we have

$$
\sum_{k=-\infty}^{0} \sum_{i=1}^{2}\left|x_{i}^{*}(k)-y_{i}^{*}(k)\right|<+\infty
$$

which implies that

$$
\lim _{n \rightarrow-\infty}\left|x_{i}^{*}(n)-y_{i}^{*}(n)\right|=0, \quad i=1,2
$$

Define $Q=\sum_{i=1}^{2} \beta_{i} Q_{i}$, where

$$
\begin{gathered}
Q_{i}=\frac{1}{m_{i}}+\frac{\tau_{i 0} a_{i 0}^{u}}{\left(k_{i}^{l}\right)^{2}}\left(1+M_{i} B_{i}^{u} a_{i i}^{u} \tau_{i j}\right)+\tau_{i j} a_{i j}^{2} \\
+\left(\tau_{i i} a_{i i}^{u}+\tau_{i j} a_{i j}^{u}\right) M_{i} B_{i}^{u} \tau_{i i} a_{i i}^{u} \\
+\tau_{i i}^{2} a_{i i}^{2}\left[A_{i}^{u}\left(\frac{A_{i 0}^{u}}{K_{i}^{l}}+A_{i i}^{u} M_{i}+A_{i j}^{u} M_{j}+c_{i}^{u}\right)\right. \\
\left.+B_{i}^{u} M_{i}\left[\frac{a_{i 0}^{u}}{\left(K_{i}^{l}\right)^{2}}+a_{i i}^{u}+a_{i j}^{u}\right]\right], \\
i, j=1,2, i \neq j .
\end{gathered}
$$

Let $\varepsilon$ be an arbitrary small positive number. It follows from (43) that there exists a positive integer $n_{1}>0$ such that $\left|x_{i}^{*}(n)-y_{i}^{*}(n)\right|<\varepsilon / Q, n<-n_{1}, i=1,2$. Therefore, for $n<-n_{1}, i, j=1,2, i \neq j$,

$$
\begin{aligned}
& V_{i 1}^{*}(n) \leq \frac{1}{m_{i}}\left|x_{i}^{*}(n)-y_{i}^{*}(n)\right| \leq \frac{1}{m_{i}} \frac{\varepsilon}{Q}, \\
& V_{i 2}^{*}(n) \leq \frac{\tau_{i 0} a_{i 0}^{u}}{\left(k_{i}^{l}\right)^{2}} \max _{p \leq n}\left|x_{i}^{*}(p)-y_{i}^{*}(p)\right| \\
& +\tau_{i j} a_{i j}^{u} \max _{p \leq n}\left|x_{j}^{*}(p)-y_{j}^{*}(p)\right| \\
& +\tau_{i i}^{2} a_{i i}^{u}\left[A_{i}^{u}\left(\frac{a_{i 0}^{u}}{k_{i}^{l}}+a_{i i}^{u} M_{i}+a_{i j}^{u} M_{j}+c_{i}^{u}\right)\right. \\
& \times \max _{p \leq n}\left|x_{i}^{*}(p)-y_{i}^{*}(p)\right| \\
& +M_{i} B_{i}^{u} \frac{a_{i 0}^{u}}{\left(k_{i}^{i}\right)^{2}} \max _{p \leq n}\left|x_{i}^{*}(p)-y_{i}^{*}(p)\right| \\
& +M_{i} B_{i}^{u} a_{i i}^{u} \max _{p \leq n}\left|x_{i}^{*}(p)-y_{i}^{*}(p)\right| \\
& \left.+M_{i} B_{i}^{u} a_{i j}^{u} \max _{p \leq n}\left|x_{j}^{*}(p)-y_{j}^{*}(p)\right|\right] \\
& \leq\left\{\frac{\tau_{i 0} a_{i 0}^{u}}{\left(k_{i}^{l}\right)^{2}}+\tau_{i j} a_{i j}^{u}\right. \\
& +\tau_{i i}^{2} a_{i i}^{u}\left[A_{i}^{u}\left(\frac{a_{i 0}^{u}}{k_{i}^{l}}+a_{i i}^{u} M_{i}+a_{i j}^{u} M_{j}+c_{i}^{u}\right)\right. \\
& +M_{i} B_{i}^{u} \frac{a_{i 0}^{u}}{\left(k_{i}^{i}\right)^{2}} \\
& \left.\left.+M_{i} B_{i}^{u} a_{i i}^{u}+M_{i} B_{i}^{u} a_{i j}^{u}\right]\right\} \frac{\varepsilon}{Q},
\end{aligned}
$$

$$
\begin{aligned}
& V_{i 3}^{*}(n) \leq M_{i} \tau_{i 0} \tau_{i i} \frac{a_{i 0}^{u} B_{i}^{u}}{\left(k_{i}^{l}\right)^{2}} a_{i i}^{u} \max _{p \leq n}\left|x_{j}^{*}(p)-y_{j}^{*}(p)\right| \\
& +M_{i} \tau_{i i}^{2} B_{i}^{u}\left(a_{i i}^{u}\right)^{2} \max _{p \leq n}\left|x_{i}^{*}(p)-y_{i}^{*}(p)\right| \\
& +M_{i} \tau_{i j} \tau_{i i} B_{i}^{u} a_{i j}^{u} a_{i i}^{u} \max _{p \leq n}\left|x_{j}^{*}(p)-y_{j}^{*}(p)\right| \\
& \leq\left\{M_{i} \tau_{i 0} \tau_{i i} \frac{a_{i 0}^{u} B_{i}^{u}}{\left(k_{i}^{l}\right)^{2}} a_{i i}^{u}+M_{i} \tau_{i i}^{2} B_{i}^{u}\left(a_{i i}^{u}\right)^{2}\right. \\
& \left.+M_{i} \tau_{i j} \tau_{i i} B_{i}^{u} a_{i j}^{u} a_{i i}^{u}\right\} \frac{\varepsilon}{Q}, \\
& i, j=1,2, i \neq j \text {. }
\end{aligned}
$$


It follows from (38) and above inequalities that

$$
V^{*}(n) \leq \sum_{i=1}^{2} \beta_{i} Q_{i} \frac{\varepsilon}{Q}=\varepsilon, \quad n<-n_{1},
$$

so $\lim _{n \rightarrow-\infty} V^{*}(n)=0$. Note that $V^{*}(n)$ is a nonincreasing function on $Z$, and then $V^{*}(n) \equiv 0$; that is $x_{i}^{*}(n)=y_{i}^{*}(n)$, $i=1,2$, for all $n \in Z$. Therefore, each hull equation of system (1) has a unique strictly positive solution.

In view of the above discussion, any hull equation of system (1) has a unique strictly positive solution. By Propositions 7-10 and Lemma 11, the almost periodic difference system (1) has a unique strictly positive almost periodic solution which is globally attractive. The proof is completed.

Let $\tau_{i j}=0, i=1,2, j=0,1,2$. Like in the proof of Theorem 12, we have the following corollary.

Corollary 13. Let $\tau_{i j}=0, i=1,2, j=0,1,2$. Assume that

$$
\begin{aligned}
& \frac{a_{10}^{l}}{M_{1}+k_{1}^{u}}-a_{12}^{u} M_{2}-c_{1}^{u}>0, \\
& \frac{a_{20}^{l}}{M_{2}+k_{2}^{u}}-a_{21}^{u} M_{1}-c_{2}^{u}>0
\end{aligned}
$$

hold and further that there exist two positive constants $\alpha_{1}$ and $\alpha_{2}$, such that

$$
\begin{array}{r}
\alpha_{i}\left[\min \left\{a_{i i}^{l}, \frac{2}{M_{i}}-a_{i i}^{u}\right\}-\frac{a_{i 0}^{u}}{\left(k_{i}^{l}\right)^{2}}\right]-\alpha_{j} a_{j i}^{u}>0, \\
i, j=1,2, i \neq j,
\end{array}
$$

where $M_{i}=\left(1 / a_{i i}^{l}\right) \exp \left\{\left(a_{i 0}^{u} / k_{i}^{l}\right)-1\right\}$. Then the almost periodic difference system (1) admits a unique strictly positive almost periodic solution, which is globally attractive.

\section{Example and Numerical Simulation}

In this section, we give the following example to check the feasibility of our result.

Example 1. Consider the following almost periodic discrete Schoener's competition model with delays

$$
\begin{aligned}
& x_{1}(n+1) \\
&=x_{1}(n) \exp \left[\frac{0.4+0.1 \sin (\sqrt{2} n)}{x_{1}(n-3)+10.2+0.2 \cos (\sqrt{2} n)}\right. \\
& \\
&-(2.02+0.02 \sin (\sqrt{3} n)) x_{1}(n-1) \\
&-(0.015+0.005 \sin (\sqrt{2} n)) x_{2}(n-4) \\
& \\
&-(0.002+0.0005 \sin (\sqrt{5} n))],
\end{aligned}
$$

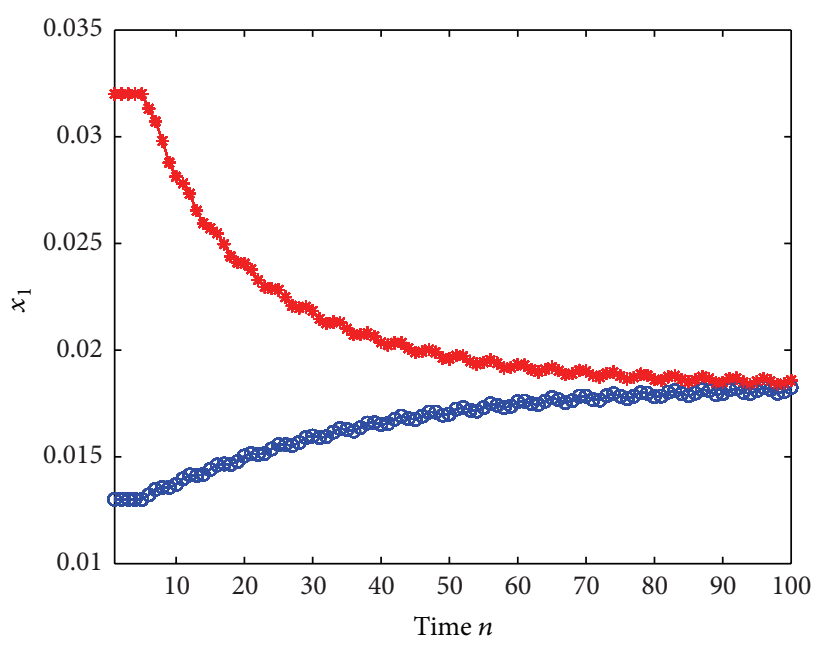

Figure 1: Dynamic behavior of the first component $x_{1}(n)$ of the solution $\left(x_{1}(n), x_{2}(n)\right)$ to system (49) with the initial conditions $\left(x_{1}(n), x_{2}(n)\right)=(0.013,0.018)$ and $(0.032,0.025), n=1,2,3,4,5$ for $n \in[1,100]$, respectively.

$$
\begin{aligned}
& x_{2}(n+1) \\
&=x_{2}(n) \exp \left[\frac{0.3+0.2 \sin (\sqrt{3} n)}{x_{2}(n-4)+10.5+0.5 \cos (\sqrt{3} n)}\right. \\
&-(0.009+0.001 \cos (\sqrt{5} n)) x_{1}(n-2) \\
&-(2.53+0.03 \cos (\sqrt{3} n)) x_{2}(n-1) \\
&-(0.005+0.001 \sin (\sqrt{2} n))]
\end{aligned}
$$

By simple computation, we derive

$$
\begin{aligned}
& M_{1} \approx 0.2033, \quad M_{2} \approx 0.1626, \\
& \frac{a_{10}^{l}}{M_{1}+k_{1}^{u}}-a_{12}^{u} M_{2}-c_{1}^{u} \approx 0.0237, \\
& \frac{a_{20}^{l}}{M_{2}+k_{2}^{u}}-a_{21}^{u} M_{1}-c_{2}^{u} \approx 0.0018, \\
& E_{11} \approx 1.1047, \quad E_{22} \approx 1.377, \\
& F_{12} \approx 1.0075, \quad F_{21} \approx 1.3366, \\
& G_{12} \approx 0.0548, \quad G_{21} \approx 0.0217 .
\end{aligned}
$$

Then

$$
\begin{aligned}
& \frac{2}{3} E_{11}-\left(\frac{2}{3} F_{12}+G_{21}\right) \approx 0.01 \\
& E_{22}-\left(F_{21}+\frac{2}{3} G_{12}\right) \approx 0.026
\end{aligned}
$$




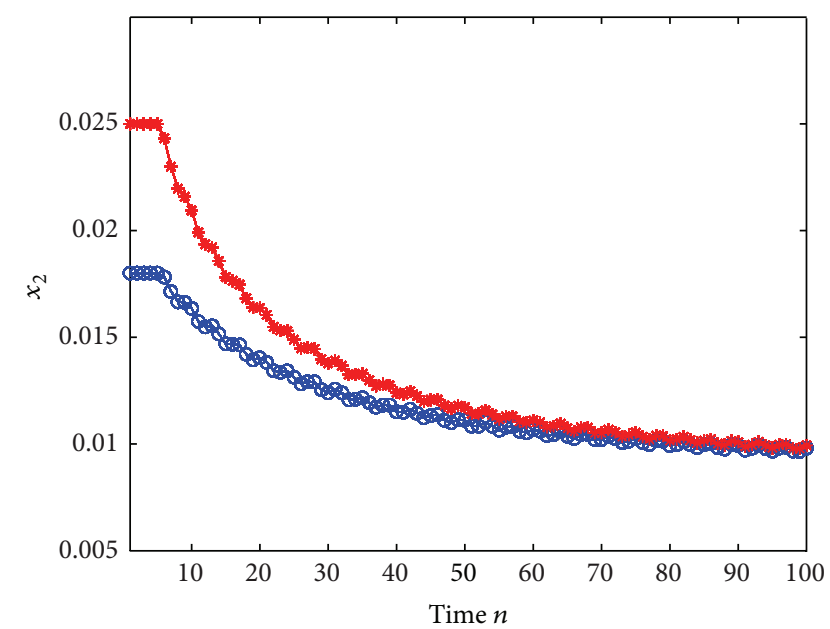

FIGURE 2: Dynamic behavior of the second component $x_{2}(n)$ of the solution $\left(x_{1}(n), x_{2}(n)\right)$ to system (49) with the initial conditions $\left(x_{1}(n), x_{2}(n)\right)=(0.013,0.018)$ and $(0.032,0.025), n=1,2,3,4,5$ for $n \in[1,100]$, respectively.

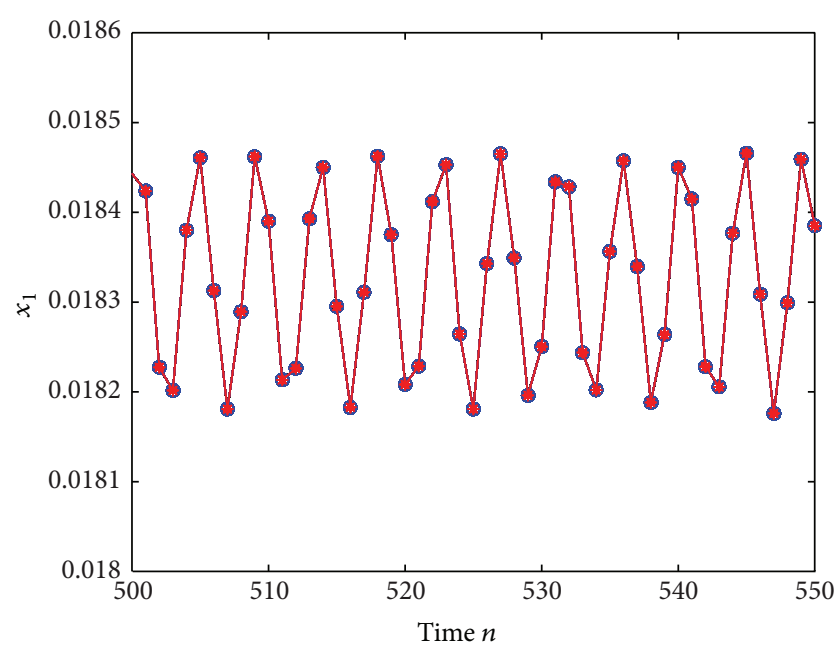

Figure 3: Dynamic behavior of the first component $x_{1}(n)$ of the solution $\left(x_{1}(n), x_{2}(n)\right)$ to system (49) with the initial conditions $\left(x_{1}(n), x_{2}(n)\right)=(0.013,0.018)$ and $(0.032,0.025), n=1,2,3,4,5$ for $n \in[500,550]$, respectively.

Also it is easy to see that conditions (H2) and (H3) are verified. Therefore, system (49) has a unique strictly positive almost periodic solution which is globally attractive. Our numerical simulations support our results (see Figures 1, 2, 3 , and 4).

\section{Conflict of Interests}

There are no financial interest conflicts between the authors and the commercial identity.

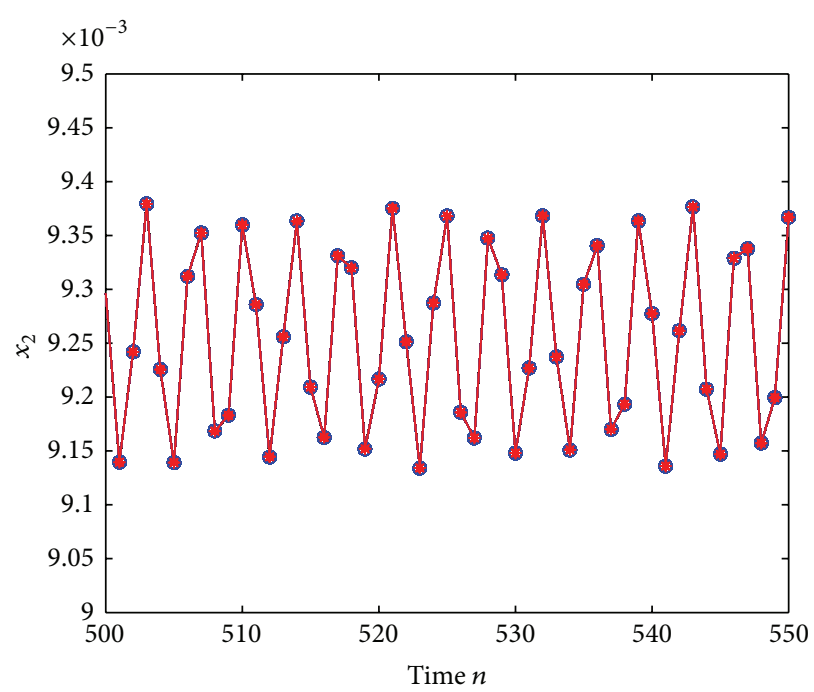

Figure 4: Dynamic behavior of the second component $x_{2}(n)$ of the solution $\left(x_{1}(n), x_{2}(n)\right)$ to system (49) with the initial conditions $\left(x_{1}(n), x_{2}(n)\right)=(0.013,0.018)$ and $(0.032,0.025), n=1,2,3,4,5$ for $n \in[500,550]$, respectively.

\section{Acknowledgments}

This work is supported by National Natural Science Foundation of China (no. 61132008) and Scientific Research Program Funded by Shaanxi Provincial Education Department of China (no. 2013JK1098).

\section{References}

[1] L. Wu, F. Chen, and Z. Li, "Permanence and global attractivity of a discrete Schoener's competition model with delays," Mathematical and Computer Modelling, vol. 49, no. 7-8, pp. 1607-1617, 2009.

[2] X. Li and W. Yang, "Permanence of a discrete $n$-species Schoener competition system with time delays and feedback controls," Advances in Difference Equations, vol. 2009, Article ID 515706, 10 pages, 2009.

[3] Q. Su and N. Zhang, "Feedback control variables have no influence on the permanence of a discrete $n$-species Schoener competition system with time delays," Discrete Dynamics in Nature and Society, vol. 2010, Article ID 583203, 12 pages, 2010.

[4] T. Zhang, Y. Li, and Y. Ye, "On the existence and stability of a unique almost periodic solution of Schoener's competition model with pure-delays and impulsive effects," Communications in Nonlinear Science and Numerical Simulation, vol. 17, no. 3, pp. 1408-1422, 2012.

[5] J. Lv, K. Wang, and M. Liu, "Dynamical properties of a stochastic two-species Schoener's competitive model," International Journal of Biomathematics, vol. 5, no. 5, Article ID 1250035, 20 pages, 2012.

[6] W. Gan and Z. Lin, "The asymptotic periodicity in a Schoener's competitive model," Applied Mathematical Modelling, vol. 36, no. 3, pp. 989-996, 2012.

[7] H. Huo and W. Li, "Existence and global stability of periodic solutions of a discrete predator-prey system with delays," 
Applied Mathematics and Computation, vol. 153, no. 2, pp. 337351, 2004.

[8] Y. Li, "Positive periodic solutions of a discrete mutualism model with time delays," International Journal of Mathematics and Mathematical Sciences, vol. 2005, no. 4, pp. 499-506, 2005.

[9] Z. Liu and L. Chen, "Positive periodic solution of a general discrete non-autonomous difference system of plankton allelopathy with delays," Journal of Computational and Applied Mathematics, vol. 197, no. 2, pp. 446-456, 2006.

[10] Y. Song and H. Tian, "Periodic and almost periodic solutions of nonlinear discrete Volterra equations with unbounded delay," Journal of Computational and Applied Mathematics, vol. 205, no. 2, pp. 859-870, 2007.

[11] S. H. Saker, "Periodic solutions, oscillation and attractivity of discrete nonlinear delay population model," Mathematical and Computer Modelling, vol. 47, no. 3-4, pp. 278-297, 2008.

[12] Y. Song, "Positive almost periodic solutions of nonlinear discrete systems with finite delay," Computers \& Mathematics with Applications, vol. 58, no. 1, pp. 128-134, 2009.

[13] J. O. Alzabut, G. T. Stamov, and E. Sermutlu, "Positive almost periodic solutions for a delay logarithmic population model," Mathematical and Computer Modelling, vol. 53, no. 1-2, pp. 161167, 2011.

[14] X. Chen and F. Chen, "Almost-periodic solutions of a delay population equation with feedback control," Nonlinear Analysis: Real World Applications, vol. 7, no. 4, pp. 559-571, 2006.

[15] X. Chen, "Almost periodic solutions of nonlinear delay population equation with feedback control," Nonlinear Analysis: Real World Applications, vol. 8, no. 1, pp. 62-72, 2007.

[16] Z. Li and F. Chen, "Almost periodic solutions of a discrete almost periodic logistic equation," Mathematical and Computer Modelling, vol. 50, no. 1-2, pp. 254-259, 2009.

[17] C. Niu and X. Chen, "Almost periodic sequence solutions of a discrete Lotka-Volterra competitive system with feedback control," Nonlinear Analysis: Real World Applications, vol. 10, no. 5, pp. 3152-3161, 2009.

[18] T. Zhang, Y. Li, and Y. Ye, "Persistence and almost periodic solutions for a discrete fishing model with feedback control," Communications in Nonlinear Science and Numerical Simulation, vol. 16, no. 3, pp. 1564-1573, 2011.

[19] Z. Li, F. Chen, and M. He, "Almost periodic solutions of a discrete Lotka-Volterra competition system with delays," Nonlinear Analysis: Real World Applications, vol. 12, no. 4, pp. 2344-2355, 2011.

[20] Y. Wang, "Periodic and almost periodic solutions of a nonlinear single species discrete model with feedback control," Applied Mathematics and Computation, vol. 219, no. 10, pp. 5480-5486, 2013.

[21] T. Zhang and X. Gan, "Almost periodic solutions for a discrete fishing model with feedback control and time delays," Communications in Nonlinear Science and Numerical Simulation, vol. 19, no. 1, pp. 150-163, 2014.

[22] A. M. Fink and G. Seifert, "Liapunov functions and almost periodic solutions for almost periodic systems," Journal of Differential Equations, vol. 5, pp. 307-313, 1969.

[23] Y. Hamaya, "Existence of an almost periodic solution in a difference equation by Liapunov functions," Nonlinear Studies, vol. 8, no. 3, pp. 373-379, 2001.

[24] S.-N. Zhang, "Existence of almost periodic solutions for difference systems," Annals of Differential Equations, vol.16, no. 2, pp. 184-206, 2000.
[25] F. Chen, "Permanence for the discrete mutualism model with time delays," Mathematical and Computer Modelling, vol. 47, no. 3-4, pp. 431-435, 2008.

[26] S. N. Zhang and G. Zheng, "Almost periodic solutions of delay difference systems," Applied Mathematics and Computation, vol. 131, no. 2-3, pp. 497-516, 2002. 


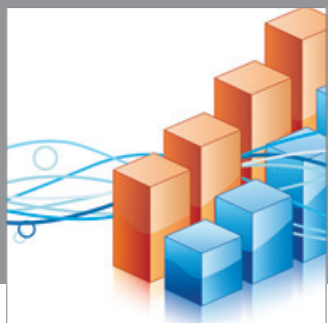

Advances in

Operations Research

mansans

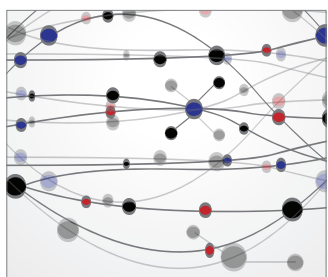

The Scientific World Journal
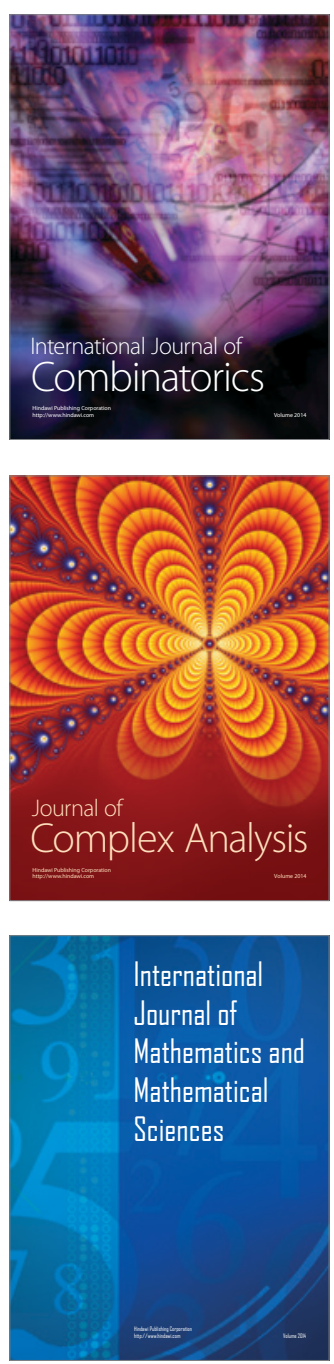
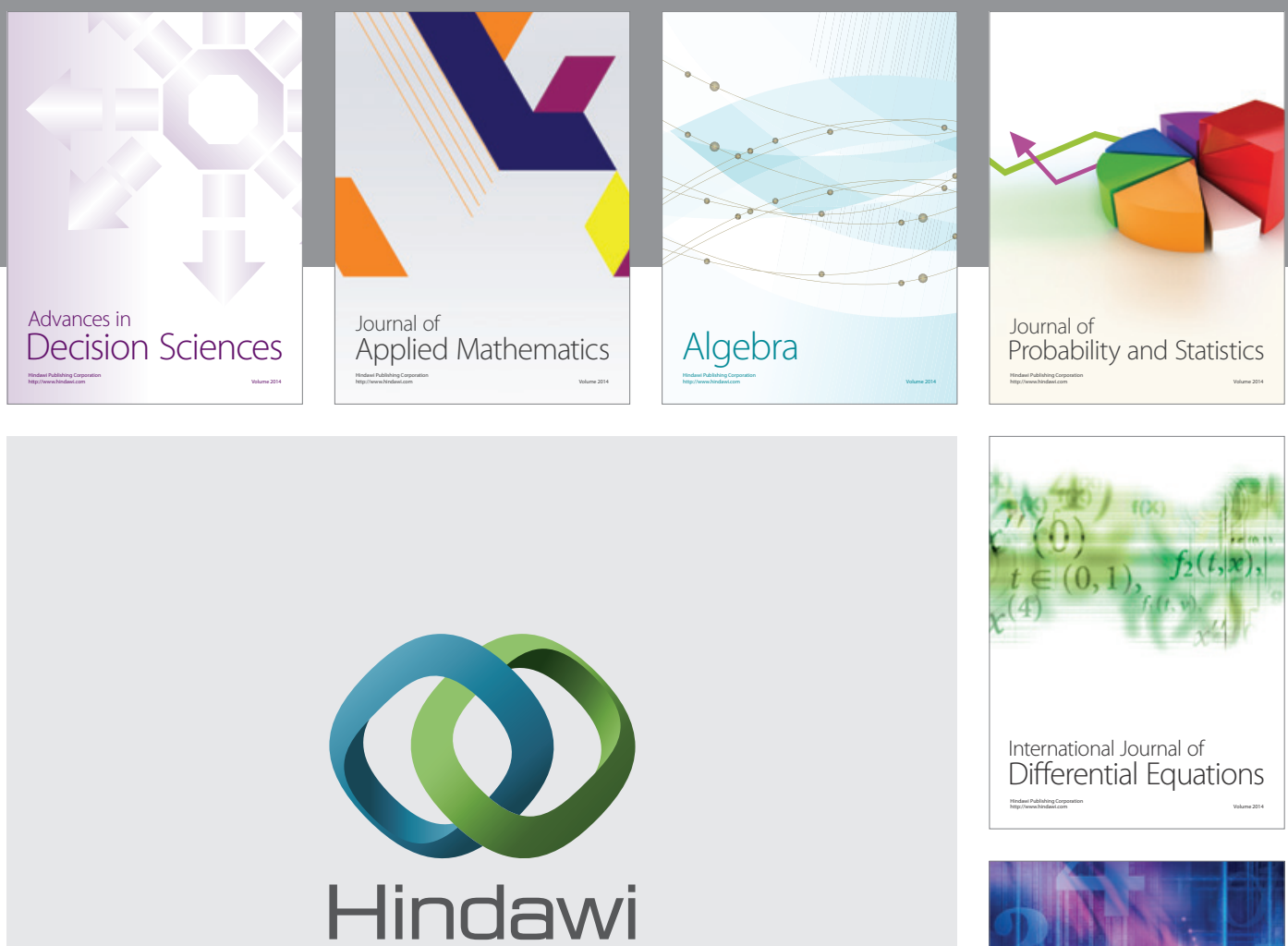

Submit your manuscripts at http://www.hindawi.com
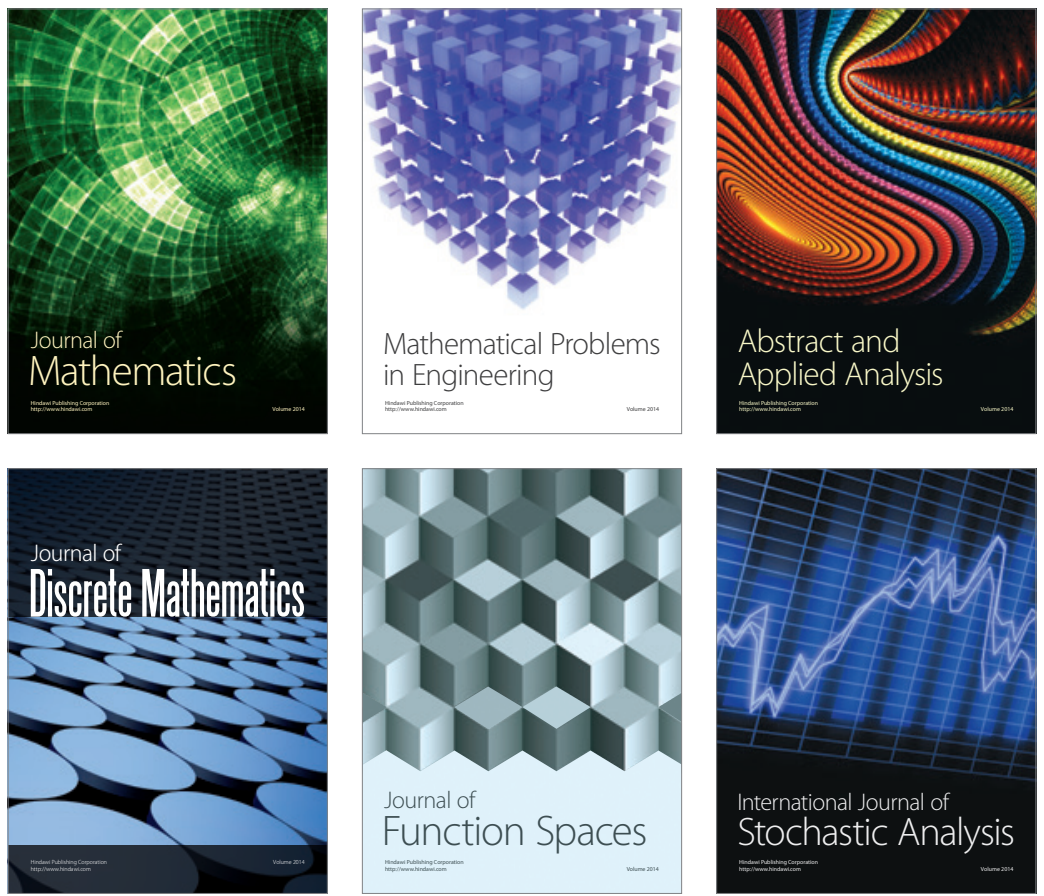

Journal of

Function Spaces

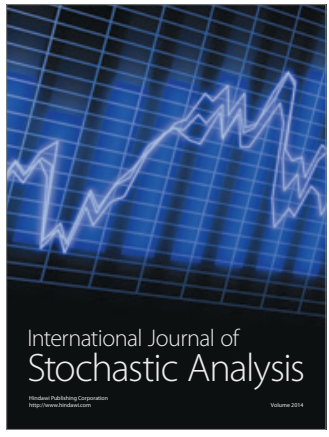

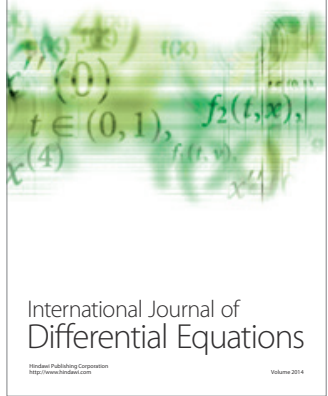
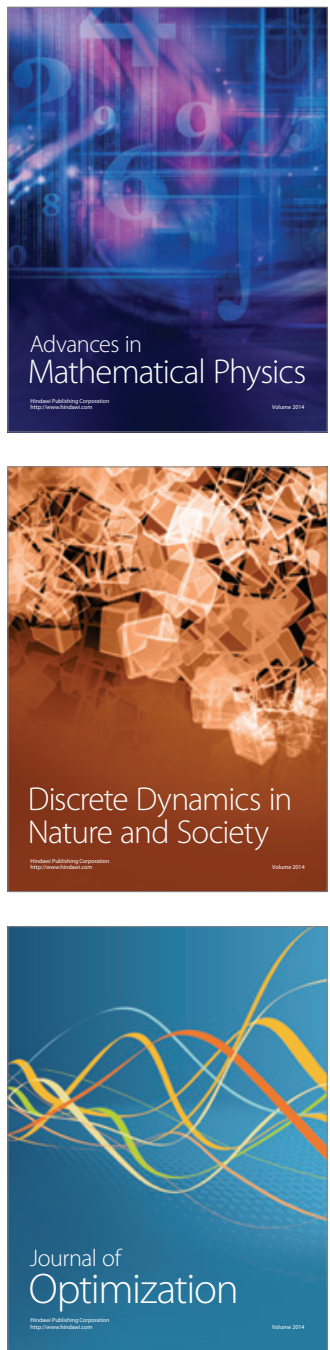